\title{
Diferenças raciais no padrão de gastos com educação: uma abordagem semiparamétrica
}

\author{
Palauras-chave \\ Curva de Engel, gasto \\ com educação, análise \\ semiparamétrica, \\ preconceito racial.
}

Classificação JEL J01, J15.

Key words

Engel's curve, education expenditure, semiparametric analysis, racial bias.

JEL Classification J01, J15.

\section{Resumo}

O presente artigo objetiva analisar o padrão de gastos com educação das famílias brasileiras, buscando identificar se há diferença racial na determinação da parcela dos gastos com esse grupo de consumo. O estudo se baseará em uma análise semiparamétrica da curva de Engel de educação, utilizando dados da Pesquisa de Orçamento Familiar (POF) - 2002/ 2003 e separando as famílias em grupos de renda com o intuito de identificar se há diferenças raciais dentro de um mesmo grupo. Os resultados sugerem que a variável "raça" é significativa para a amostra como um todo, mas demonstra ser significante (a 5\% de confiança) para apenas o último grupo analisado, indicando que, entre os mais pobres, não há diferença racial na decisão de dispêndio com educação.
Pollyana Jucá Santana Doutoranda da UFPE

Tatiane Almeida de Menezes Professora da UFPE

\section{Abstract}

This papers analyzes the pattern of expenditure by Brazilian families on education, looking for to identify whether there is racial difference in the determination of the type of expenses. The study is based on a semi-parametric analysis of the Engel curve of education, using data from the Research of Family Budgets (POF) - 2002/2003 and separating the families into income groups to identify whether there are racial differences inside a same group. The results suggest that the variable "race" is significant for the sample as a whole, but it becomes significant (5\% of trust) only for the last group, indicating that there is no racial difference in the expenditure decision with education among the poorest families. 


\section{1_Introdução}

A estabilização da economia brasileira, após o Plano Real, proporcionou aumento da renda média e melhoria nos gastos dos brasileiros. Trabalhos como os de Hoffman (2000 e 2006); Soares (2000, 2006a e 2006b) e Ferreira (2000), Ferreira et al. (2006) mostram de fato uma redução na desigualdade de renda e uma concomitante diminuição da pobreza após 1996. Entretanto, redução da desigualdade, embora fundamental, não implica necessariamente maior inclusão social. Como sugere Hoffmann (2000 e 2006), a qualidade do perfil dos gastos é fundamental para se perceber seu impacto sobre o bem-estar.

Segundo Ferreira (2000), as causas de uma distribuição desigual de renda devem pertencer a pelo menos um dos cinco grupos de fatores distintos relacionados a seguir: características individuais natas (raça, gênero, inteligência e riqueza inicial), características individuais adquiridas (educação, experiência), mecanismos utilizados pelo mercado para diferenciar níveis salariais (discriminação, segmentação e projeção), imperfeições no mercado de capitais (crédito e seguros) e questões demográficas de formação do domicílio. Uma das características mais importantes desses cinco grupos é a educação, que, de acordo com Schwartzman (2004), tem sido apresentada como o principal instrumento para solucionar os problemas de pobreza, desigualdade e falta de oportunidade que afetam os segmentos mais pobres da população.

Autores como Soares (2000) e Marinho e Nogueira (2006) tentaram identificar se a distribuição desigual da renda entre a população branca e negra brasileira tem algum componente de discriminação. De fato, quando separamos em grupos étnicos, negros/pardos representam 55\% da população brasileira e possuem renda total domiciliar per capita média (mensal) de $\mathrm{R} \$ 409,00$, ao passo que brancos auferem em média $\mathrm{R} \$ 801,00$ (POF 2002/03, valores correntes de 2003). Os autores citados argumentam que, se a diferença salarial se deve apenas à diferença no nível educacional, a tese da discriminação fica enfraquecida. Ou seja, se brancos e negros com mesmo nível educacional recebem a mesma renda, provavelmente não deve haver discriminação no mercado do trabalho.

Soares (2000) encontra que os homens negros mais pobres pouco teriam a ganhar com o fim da discriminação - algo em torno de $5 \%$ a $7 \%$; já os homens negros mais ricos sofreriam redução de $27 \%$ no salário que receberiam se enfrentassem a mesma estrutura salarial dos homens brancos, ou seja, esse autor constata diferenças 
de discriminação entre as faixas de renda. Utilizando uma metodologia semiparamétrica (estimador de núcleo ponderado), Marinho e Nogueira (2006) mostra, com base em simulações, que a desigualdade salarial entre raças é causada principalmente por diferenças na educação, e não por preconceito racial, ou seja, negros/pardos teriam salários menores no mercado de trabalho por possuírem nível educacional menor que os brancos, não encontrando discriminação racial.

Por esses resultados, temos que a diferença educacional entre as duas raças citadas é importante no diferencial de salários, e, nesse contexto, este trabalho investiga se há desigualdade (para não repetir "diferenças") no padrão de gastos com educação entre famílias brancas e negras/pardas. $\mathrm{O}$ principal objetivo é identificar se os chefes de família de (diferentes) raças distintas apresentam um diferente perfil de gastos com educação. Uma vez encontrado que os chefes de família brancos gastam mais em educação do que os negros/pardos, é esperado que os primeiros venham a ter maior nível educacional e consequentemente maior remuneração no futuro. Caso contrário, a discriminação teria um peso na determinação da desigualdade de rendimento entre raças.

$\mathrm{Na}$ literatura internacional, estudos dos determinantes dos gastos são feitos com base na estimação da curva de Engel (Blundell et al., 1998; Bhalotra e Attfield, 1998; Gong et al., 2000; Blundell et al., 2003; Blundell et al., 2007). Nesse contexto, o presente artigo busca examinar como as famílias alocam seu orçamento, observando qual a parcela é destinada a gastos com educação, e que, além da renda, outros fatores são de suma importância na alocação do orçamento familiar, como características do chefe da família, composição do domicílio e localização desse.

A forma mais usual de estimação da curva de Engel é a utilização do modelo linear paramétrico descrito por Deaton e Muellbauer (1980) e conhecido por Piglog. Entretanto, como não há consenso sobre o formato da curva de Engel, a fim de evitar a má especificação dela, diferentes alternativas de estimação também têm sido utilizadas, como o modelo Tobit e os métodos não paramétricos e semiparamétricos. Alves (2000) e Kingdon (2005) utilizam o primeiro método para estimação da curva de Engel para gastos com saúde da cidade de São Paulo e gastos com educação na Índia, respectivamente, mas esse modelo é inconsistente na presença de erros heteroscedásticos.

Estimando um modelo linear e outro semiparamétrico, Blundell et al. (1998) analisa diferença de gastos entre casais com um filho e casais com dois filhos e encon- 
tra que o formato linear ajusta bem somente os dados de alimentação do Reino Unido. Bhalotra e Attfiel, (1998) ajusta o modelo linear e outro semiparamétrico para dados do Paquistão com o objetivo de identificar se há preconceito sexual na determinação da parcela da renda destinada a cada grupo de bens. O modelo semiparamétrico também é utilizado por Gong et al. (2000), com o objetivo de identificar se há diferenciação nos gastos familiares entre famílias que têm filhos do sexo masculino e feminino na China.

Todos esses modelos semiparamétricos consideram que a parcela dos gastos de determinado bem se relaciona de forma não linear apenas com os gastos totais, mas de maneira linear com os demais regressores. Recentemente, Blundell et al. (2003 e 2007) desenvolveram um algoritmo que permite estimar a função demanda, inserindo na parte não linear não apenas a renda, mas também as características familiares. Tal algoritmo tem por principal finalidade resolver o problema de heterogeneidade entre os diversos gastos individuais, na estimação da função demanda. No presente trabalho, uma vez que a parcela dos gastos de interesse é apenas a dos gastos em educação, optou-se por seguir a metodologia desenvolvida por Robinson (1988) e a desenvolvida por Bhalotra e Attfield (1998) e Gong et al. (2000) para estimação da curva de Engel.
Este trabalho tem por objetivo identificar se existem diferenças no padrão de gastos com educação entre chefes de famílias brancas e negras; para tanto, seguindo a metodologia mais empregada na literatura (Bhalotra e Attfield, 1998; Gong et al., 2000), aplica-se um modelo semiparamétrico para estimação da curva de Engel e, em seguida, observa-se o impacto da raça sobre os gastos com educação. Uma vez encontrado que não há diferença no padrão dos gastos com educação das famílias para chefes de ambas as raças, fica reforçada a tese de que existe discriminação no mercado de trabalho. Caso contrário, o diferencial no padrão de gastos com educação entre brancos e negros pode ser uma das causas do diferencial de salários, enfraquecendo assim a tese da discriminação.

Para desenvolver o presente estudo, trabalha-se com duas distintas definições de gastos em educação. Na primeira, determina-se (Para não repetir “define-se") gastos com educação, da mesma forma que Castro e Vaz (2007), incluindo todos os gastos contidos no grupo Educação do IBGE (ver tabela do Anexo). A segunda definição de gastos com educação assume que esses são compostos apenas de mensalidades escolares (ensino fundamental, médio e superior), material escolar e livros didáticos.

Além desta introdução, há mais quatro seções neste artigo. A segunda seção 
1 Alem

1 Além das pessoas que se declararam filhos da pessoa de referência do domicílio, consideramos outra categoria, "demais parentes", uma vez que pode haver netos, sobrinhos, enteados que são de responsabilidade do chefe do domicílio, não sendo seu filho legítimo.

2 Ver no Anexo os itens que compõem os grupos, sendo a classificação de Castro e Vaz (2007). descreve e analisa os dados empregados no trabalho; a terceira seção expõe a metodologia proposta; a quarta apresenta os resultados obtidos e, por fim, as conclusões deste trabalho se encontram na seção cinco.

\section{Dados}

Os dados utilizados neste trabalho são da Pesquisa de Orçamento Familiar (POF) realizada pelo Instituto Brasileiro de Geografia e Estatística (IBGE) no período compreendido entre julho de 2002 e junho de 2003. A amostra consiste em 48.568 domicílios de todo o País, e a média de pessoas por domicílio é de 3,75. Como buscamos identificar diferenças raciais nos gastos com educação, limitamos nossa amostra a domicílios que tivessem filhos ${ }^{1}$ entre seis e 25 anos e que o chefe da família se declarou branco, negro ou pardo, agregando negros e pardos, o que resultou num total de 29.864 domicílios.

A POF 2002/03 do IBGE apresenta 216 itens de gastos com educação, agrupados em cinco subgrupos: cursos regulares, outros cursos, livros didáticos, artigos escolares, taxas e outros gastos (para maiores detalhes ver Castro e Vaz, 2007). ${ }^{2}$ Optou-se aqui por trabalhar com duas definições de educação: na primeira, gastos com educação são definidos como todos aqueles que possuem impacto direto e indireto na formação de um indivíduo. A ideia aqui defendida é que o dispêndio com uniforme, material escolar, taxas de bibliotecas, aulas de reforço, cursos técnicos, aulas de esporte, curso pré-vestibular, entre outros, é um gasto que contribui direta ou indiretamente para o melhor desempenho dos estudantes, estando assim colaborando para o desenvolvimento educacional da criança/jovem. Nesse caso, todos os itens que compõem o grupo Educação do IBGE estão compondo os gastos com educação, estando no artigo denominado de "gastos globais com educação".

A segunda definição é mais restrita e só considera gastos com educação as despesas com cursos regulares (ensino fundamental, médio e superior), livros didáticos e material escolar. Considerando nesse caso os gastos que estão diretamente relacionados com a frequência escolar, sendo no restante do texto, denominados de "gastos diretos". A Tabela 4 descreve o percentual dos gastos familiares com educação, por faixa de renda, para as duas definições acima.

Dividindo a amostra em quatro estratos de renda com aproximadamente o mesmo número de famílias em cada um, é possível analisar se em grupos homogêneos em relação à renda há diferenças étnicas nos gastos com educação. Na Tabela 1, encontram-se as características do chefe da família e número de filhos do domicílio. 
Tabela 1_Estatísticas sobre o chefe da família

\begin{tabular}{|c|c|c|c|c|c|c|}
\hline & \multicolumn{2}{|c|}{ \% de famílias na faixa } & \multicolumn{2}{|c|}{$\begin{array}{l}\text { Anos de estudo } \\
\text { do chefe do domicílio }\end{array}$} & \multicolumn{2}{|c|}{ Número de filhos } \\
\hline & Brancos & $\begin{array}{l}\text { Negros/ } \\
\text { Pardos }\end{array}$ & Brancos & $\begin{array}{l}\text { Negros/ } \\
\text { Pardos }\end{array}$ & Brancos & $\begin{array}{c}\text { Negros/ } \\
\text { Pardos }\end{array}$ \\
\hline Faixa 1 & 26,31 & 73,69 & 3,51 & 2,74 & 2,43 & 2,79 \\
\hline Faixa 2 & 36,01 & 63,99 & 4,44 & 3,69 & 2,02 & 2,24 \\
\hline Faixa 3 & 46,53 & 53,47 & 5,72 & 5,33 & 1,86 & 2,01 \\
\hline Faixa 4 & 61,76 & 38,24 & 9,66 & 8,33 & 1,69 & 1,77 \\
\hline Total & 41,61 & 58,39 & 6,73 & 4,63 & 1,90 & 2,26 \\
\hline
\end{tabular}

Fonte: Elaboração própria a partir da POF/IBGE.

Tabela 2_Renda monetária média domiciliar "per capita" por faixa de renda1

\begin{tabular}{|c|c|c|c|}
\hline & Brasil & Brancos & Negros/ Pardos \\
\hline Faixa 1 & 44,75 & 45,89 & 44,23 \\
\hline Faixa 2 & 123,49 & 126,25 & 121,47 \\
\hline Faixa 3 & 256,59 & 259,87 & 252,79 \\
\hline Faixa 4 & $1.030,41$ & $1.101,58$ & 863,41 \\
\hline Total & 400,80 & 536,28 & 260,39 \\
\hline
\end{tabular}

(1) (Em R $\$$ e mensal). Como a pesquisa foi realizada ao longo de um ano, o IBGE deflacionou todos os valores de renda

e gastos para 15 de janeiro de 2003, utilizando diversos indexadores em relação aos gastos. Neste trabalho utilizaremos

somente renda e gastos monetários.

Fonte: Elaboração própria a partir da POF/IBGE.

Tabela 3_Gasto monetário domiciliar "per capita" médio (R\$)

\begin{tabular}{|c|c|c|c|}
\hline & Brasil & Brancos & Negros/Pardos \\
\hline Faixa 1 & 93,29 & 109,31 & 86,03 \\
\hline Faixa 2 & 162,80 & 173,46 & 155,03 \\
\hline Faixa 3 & 294,16 & 311,87 & 273,61 \\
\hline Faixa 4 & 959,53 & $1.049,95$ & 747,36 \\
\hline Total & 410,15 & 548,06 & 267,22 \\
\hline
\end{tabular}

Fonte: Elaboração própria a partir da POF/IBGE. 
Tabela 4_Porcentagem do gasto monetário domiciliar com educação

\begin{tabular}{|c|c|c|c|c|c|c|}
\hline & Brasil & Brancos & $\begin{array}{c}\text { Negros/ } \\
\text { Pardos }\end{array}$ & Brasil & Brancos & $\begin{array}{l}\text { Negros, } \\
\text { Pardos }\end{array}$ \\
\hline Faixa 1 & 1,21 & 1,29 & 1,18 & 1,47 & 1,56 & 1,42 \\
\hline Faixa 2 & 1,17 & 1,16 & 1,17 & 1,56 & 1,59 & 1,54 \\
\hline Faixa 3 & 1,54 & 1,53 & 1,55 & 2,25 & 2,27 & 2,22 \\
\hline Faixa 4 & 3,95 & 4,35 & 3,01 & 5,36 & 5,83 & 4,25 \\
\hline Total & 2,07 & 2,53 & 1,59 & 2,81 & 3,44 & 2,15 \\
\hline
\end{tabular}

Fonte: Elaboração própria a partir da POF/IBGE.

As familias negras/pardas representam 58,39\% das familias brasileiras na amostra, e essas se concentram nas faixas mais baixas de renda. Em relação à média de anos de estudo, observa-se que a diferença no nível educacional do chefe da família entre as faixas de renda é alta, sendo superior para brancos em todas as faixas e chegando a mais de dois anos para a população total. Por fim, em relação ao número de filhos, observa-se que a média cai à medida que a renda aumenta para ambas as raças, mas deve-se ressaltar que, apesar da diferença não ser muito grande, famílias chefiadas por pessoas brancas possuem uma média menor.

Como mencionado na introdução, nota-se nos dados uma grande diferença na renda e também nos gastos médios entre as famílias dos dois grupos considerados. É possível constatar tal diferença pelos dados contidos na Tabela 2, que apresenta a renda familiar per capita média, e na ferramenta não paramétrica para análise de dis- tribuições, a função de densidade da renda que se encontra na Figura 1.

Em relação à renda monetária média domiciliar per capita, observamos que somente entre os $25 \%$ mais ricos a diferença entre brancos e não brancos é significativa; para as demais faixas, a renda é muito similar entre os dois grupos. A renda per capita média de negros/pardos é muito inferior a dos brancos $(\mathrm{R} \$ 260,00$ contra $\mathrm{R} \$ 536,00)$, já que, além dessa grande diferença entre os mais ricos, nas camadas mais baixas há maior número de famílias negras/pardas.

Esse fato é confirmado na Figura 1 pela função de densidade do logaritmo da renda, na qual a média de renda dos negros/pardos (o pico da distribuição) é deslocada para a esquerda, ou seja, há uma concentração muito menor de pessoas na cauda superior da distribuição e uma grande concentração de pessoas na média (o pico é mais elevado). 
Figura 1_ Função densidade da renda monetária domiciliar "per capita", dados da POF 2002-03

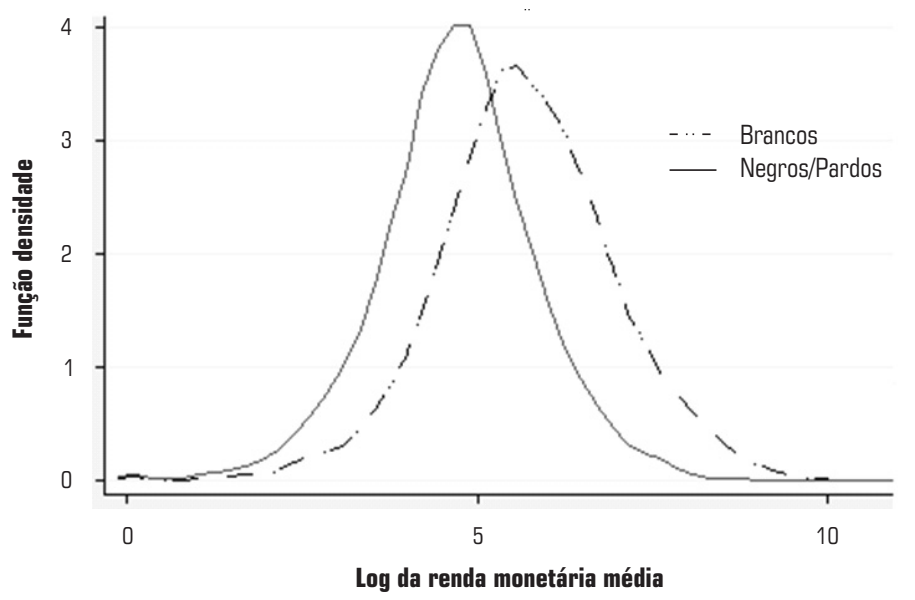

Fonte: Elaboração própria a partir da POF/IBGE.

Já a análise de dominância estocástica de segunda ordem é uma ferramenta não paramétrica que compara duas ou mais distribuições de uma mesma variável, buscando identificar se alguma delas é dominante em relação às outras. Uma distribuição é dita dominante estocasticamente de segunda ordem se:

$D_{2}(y)=\int^{x} F_{2}(t) d t \quad \geq \int^{x} F_{1}(t) d t=D_{1}(y)$

ou

$F_{2}(y) \geq F_{1}(y)$

para qualquer ponto de distribuição
Ou seja, a distribuição de renda dos brancos será considerada dominante em relação à distribuição dos não brancos se a renda dos primeiros for mais elevada em todos os pontos da distribuição; em outras palavras, se houver mais pobres entre os negros/pardos. Confirmamos essa dominância pela Figura 2, onde a distribuição dos brancos se encontra abaixo da distribuição dos não negros/pardos; elas só se interceptam, mas não se cruzam, nas últimas faixas de renda. Isso significa que, em todos os pontos da distribuição, há mais pobres entres negros/pardos do que entre a outra raça. 
Figura 2_ Dominância estocástica de segunda ordem, dados da POF 2002-03

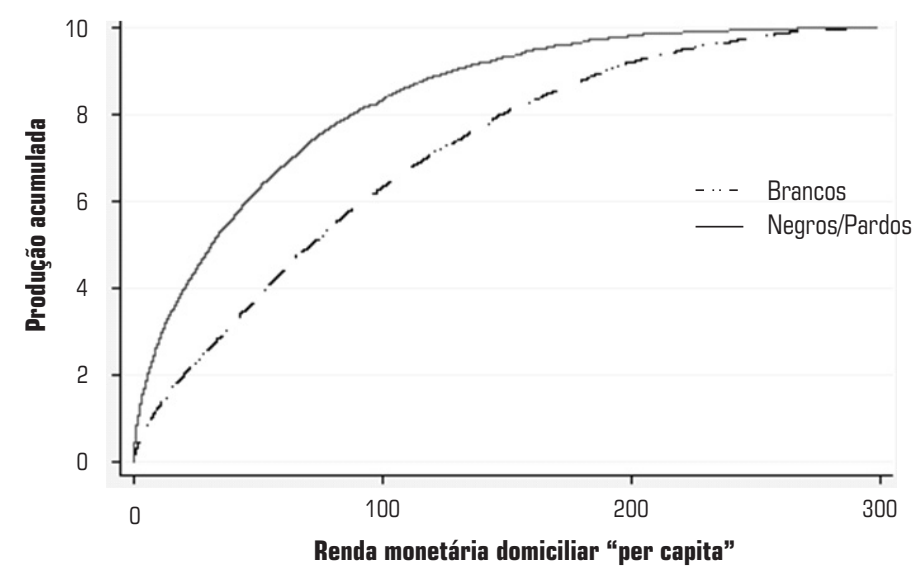

Fonte: Elaboração própria a partir da POF/IBGE.

A renda média é mais elevada para brancos, porém mais concentrada. A curva de Lorenz nos mostra a relação entre os percentis da população e os percentis da renda. Caso a renda fosse igualmente distribuída, a curva de Lorenz seria uma reta de $45^{\circ}$; logo, quanto mais concentrada uma distribuição, maior sua curvatura. $\mathrm{O}$ índice de Gini da renda para a população total é de 0,58 , para brancos esse valor é de 0,57 , e para o outro grupo é de 0,55 . Essa maior concentração se dá em toda a distribuição, e, em todas as faixas de renda, a distribuição é menos desigual para negros/pardos, como pode ser observada pela Figura 3.

É possível constatar essas mesmas diferenças raciais no comportamento dos gastos das famílias brasileiras. Como pode ser observado na Tabela 3 , essa variável segue o mesmo padrão da renda e apresenta uma média maior para brancos do que para negros/pardos em todas as faixas de renda. Apesar de possuírem a mesma renda média per capita (famílias dentro de uma determinada faixa), as famílias se comportam de forma distinta em relação ao seu dispêndio, tendo algumas que gastam muito mais do ganham. Os gastos superam a renda em quase todas as faixas, tanto para brancos quanto para não brancos. Os 10\% mais ricos são os únicos que possuem renda média domiciliar per capita maior que gastos domiciliares médios per capita, e essa diferença é maior para negros/pardos. 
Figura 3_ Curva de Lorenz da renda monetária domiciliar "per capita", dados da POF 2002-03

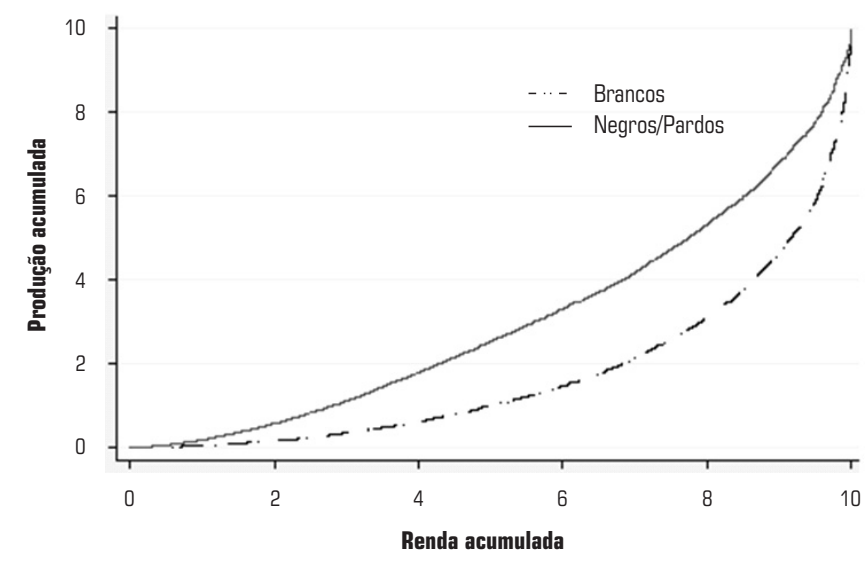

Fonte: Elaboração própria a partir da POF/IBGE.

Apesar de a média de gastos ser maior gastos (Figura 4) demonstra que essa difepara brancos, a função de densidade dos rença é menor do que a renda (ver Figura 1).

\section{Figura 4_ Função densidade do gasto monetário domiciliar "per capita",} dados da POF 2002-03

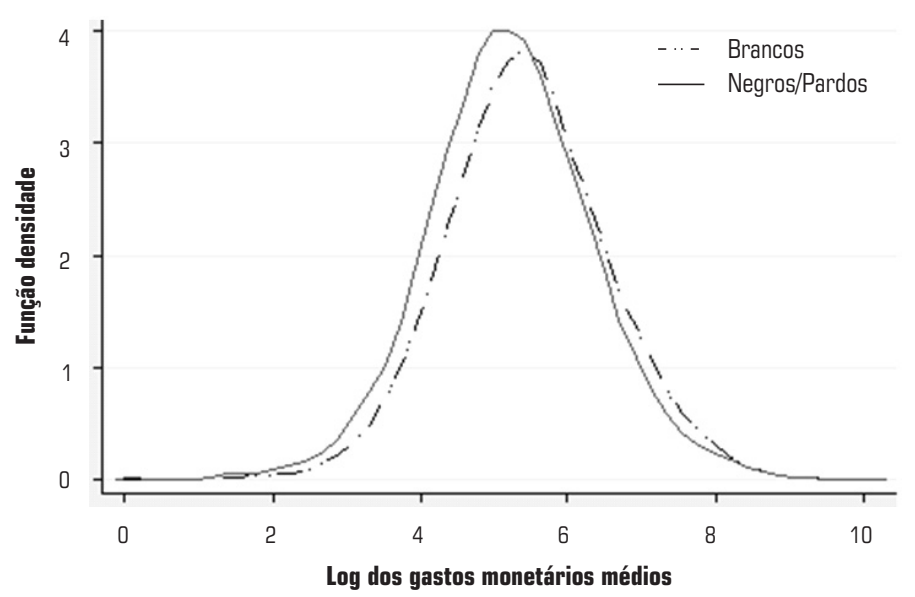

Fonte: Elaboração própria a partir da POF/IBGE. 
Nota-se que, em relação aos gastos, também há uma grande concentração para os brancos, ou seja, a curva de Lorenz para esse grupo apresenta maior curvatura, e o índice de Gini dos gastos per capita é de 0,57 (para negros/pardos, o índice é de 0,55).

Apesar de apresentarem tanto renda quanto gastos maiores, familias com chefe da raça branca não gastam significativamente mais com educação do que famílias não brancas; para algumas faixas de renda, negros/pardos até gastam mais do que o outro grupo (veja Tabela 4). Dessa forma, não é possível concluir se chefes de família de determinada raça possuem maior dispêndio com educação do que a outra; por isso se realiza uma análise da curva de Engel para os gastos com educação, avaliando a elasticidade-gasto para esse grupo de despesa. $\mathrm{Na}$ Tabela 4, temos a porcentagem dos gastos com educação (total e diretos).

\section{3_Metodologia}

A curva de Engel é derivada de uma função demanda e mensura como varia a quantidade demandada de determinado bem graças a uma variação na renda. Entretanto, como são disponibilizadas somente informações dos gastos e não da quantidade demanda de cada bem, precisamos fazer uma análise de gastos ao invés de uma análise de demanda. Considerando uma restrição orçamentária linear, a forma geral da curva de Engel será:

\section{Figura 5_Curva de Lorenz do gasto domiciliar "per capita",} dados da POF 2002-03

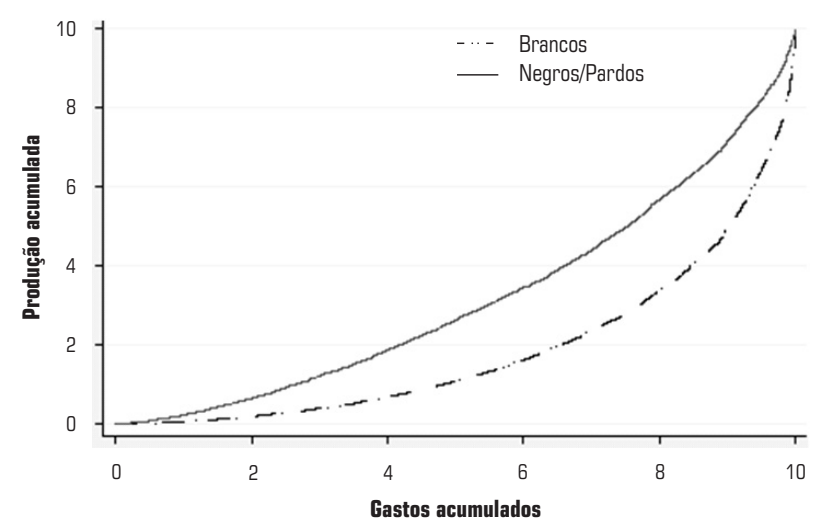

Fonte: Elaboração própria a partir da POF/IBGE. 
$w_{i}=f_{i}(g)$

Onde $w_{i}$ é a parcela dos gastos com um determinado bem em relação aos gastos totais, e $(g)$ é o gasto total. Uma vez que todas as famílias se deparam com os mesmos preços na economia, estudos da curva de Engel assim como estudos de demanda utilizam técnicas de agregação, e, ao empregar dados de corte transversal, é possível inserir características individuais na estimação. Desta forma, um vetor $(x)$ é inserido na equação (2.1), que representará as características individuais relevantes na determinação de $w_{i}$. A forma linear de (2.1) foi estimada pela primeira vez por Working em 1943 e utilizada por Leser em 1963, em que a parcela dos gastos se relacionaria linearmente com o logaritmo da renda (Deaton e Muellbauer, 1980b):

$w_{i}=f_{i}(\log (g), x)$

O modelo econométrico para a equação (2.2) pode ser derivado do modelo de demanda de Deaton e Muellbauer (1980) (Almost Ideal Demand System - AIDS), ou de um modelo Indireto Translog, ambos denominados de modelo "Piglog", em que a função $f_{i}$ é linear e é a mesma para todas as famílias:

$w_{i}=\alpha_{i}+\gamma_{i} \ln g+\beta_{1}^{\prime} x+\varepsilon_{i}$

$\operatorname{com} E\left(\boldsymbol{\varepsilon}_{i} \mid \ln g\right)=0$
O problema em estimar (2.3) é que essa forma funcional é muito restritiva, como $\sum w_{i}=1$; se for adotada a hipótese de linearidade para um determinado bem, terá que se adotar tal hipótese para todos os bens. Apesar dessa limitação, o modelo AIDS tem sido amplamente empregado na estimação da função demanda global, como pode ser visto nos trabalhos de Alves et al. (1982), Michalek e Keyzer (1992) e Menezes et al. (2008).

A fim de contornar o problema da não linearidade dos gastos, alguns autores optaram por estimações não paramétricas. Blundell et al. (1998), Bhalotra e Attfield (1998) e Gong et al. (2000) empregam tal metodologia e argumentam que a estimação não paramétrica é inviável para amostras finitas e com um grande número de variáveis explicativas, sugerindo métodos semiparamétricos na estimação da curva de Engel. Os dois últimos autores optam por empregar um modelo linear semiparamétrico como proposto por Robinson (1988). Neste, a variável dependente se relaciona de forma não linear com apenas um regressor, com os gastos totais e de maneira linear com os demais (características demográficas e geográficas das famílias), como pode ser observado na equação (2.4) abaixo:

$w_{i}=\beta_{i}^{\prime} x+F_{i}(\ln g)+\varepsilon_{i}$ 
A função desconhecida $F_{i}$ representa a parte não paramétrica, que relaciona a parcela dos gastos de um determinado bem com os gastos totais, e, por ser um vetor de dimensão $(\mathrm{N} \times 1)$ de uma única variável, evita o problema típico da estimação não paramétrica, que é a presença de vários regressores. Por sua vez, a análise sobre a importância das características familiares na determinação da parcela dos gastos é feita com base nos parâmetros da parte linear.

Além do problema da não linearidade dos gastos, a estimação que utiliza dados de corte transversal valendo-se de pesquisas familiares apresenta pelo menos dois problemas econométricos que devem ser considerados no momento de estabelecer a metodologia. O primeiro é a existência de dados censurados, já que, das 29.864 famílias da amostra, 24\% declararam ter gasto nulo com educação, apesar de todas elas terem filhos em idade escolar. Nesse caso, gasto zero com educação pode estar representando uma preferência por escolas públicas, o que geraria o problema de censura nos dados e endogeneidade dos regressores. Outra fonte de endogeneidade observada é o fato de a parcela dos gastos com educação e os gastos totais serem determinados simultaneamente, de modo que $E(\varepsilon i \mid \ln g) \neq 0$. Ambos os problemas tornam o estimador de Mínimos Quadrados Ordinários (MQO) não consistente. Uma alternativa para solucionar o problema da censura é a estimação do modelo Tobit, como empregado por Kingdon (2005) e Alves (2000); essa alternativa, entretanto, não resolve o problema da endogeneidade dos gastos. A opção encontrada foi utilizar o modelo semiparamétrico linear, empregando a renda monetária para instrumentalizar os gastos totais (ver Bhalotra e Attfield (1998) e Gong et al. (2000)).

Neste caso, a estimação de (2.4) darse-á em 3 etapas. Primeiro deve ser calculada de forma não paramétrica a esperança condicional da variável dependente $\left(w_{i}\right)$ em relação ao logaritmo dos gastos monetários familiares totais $E\left(w_{i} \mid \ln g\right)$, assim como a esperança condicional das variáveis explicativas (vetor $x$ ) em relação à $\ln (g): E\left(x_{i} \mid \ln g\right)$.

Segundo Rozelle e Gibson (2000), uma regressão não paramétrica calcula a função, $m(x)=E(y \mid x)$, computando um estimador local de $y$ dentro de uma faixa específica de $x$. Se o estimador mantém fixo o tamanho da banda, conhecido como Kernel, conserva-se constante o número de observações em cada faixa e é chamado de "estimador de vizinhança" (Lowess). O problema com o primeiro é que ele pode não se apresentar robusto, uma vez que as bandas de tamanho fixo terão poucas observações na cauda superior, e a distribuição de renda apresenta tal comportamento. A equação (2.4) então se tornará: 
$E\left(w_{i} \mid \ln g\right)=\beta_{i}^{\prime} E(x \mid \ln g)+F_{i}(\ln g)$

Após estimar as esperanças condicionais da equação (2.6) pelo método Lowess, o segundo passo é subtrair (2.6) de (2.4):

$w_{i}-E\left(w_{i} \mid \ln g\right)=\beta_{i}^{\prime}(x-E(x \mid \ln g))+\varepsilon_{i}$

$w_{i}^{*}=\beta_{i}^{\prime} x_{i}^{*}+\varepsilon_{i}$

Onde as variáveis com (*) já foram transformadas pelo processo explicado anteriormente. Por fim, a última etapa é estimar de forma linear a equação (2.8):

$$
\begin{aligned}
& w_{i}^{*}=\alpha+\beta_{1} \text { filhos }^{*}+\beta_{2} \text { raça }^{*}+\beta_{3 \text { gênero }}+ \\
& +\beta_{4} e s c^{*}+\beta_{5 n e^{*}}+\beta_{6} c^{*}+\beta_{7 s u^{*}}+ \\
& +\beta_{8} n 0^{*}+\varepsilon_{i}
\end{aligned}
$$

Em que a variável dependente $\left(w_{i}^{*}\right)$ representa a parcela de gastos com educação transformada; filhos representa o número de filhos do domicílio; raça é uma dummy que assume o valor 1 se o chefe da família se declarar branco, 0 em caso contrário; gênero é uma dummy que assume valor 1 se o chefe da família for do sexo masculino, e 0 se for mulher; esc representa o número de anos de estudo do chefe da família variando entre 0 e 16, e as últimas quatro variáveis são dummies de região, sendo a região Norte usada como base, visto que é a que possui menor média de gasto com educação. Seguindo Blundell et al. (2007) e Gong et al. (2000), será utilizada a renda monetária como instrumento, em que no primeiro estágio faz-se a regressão dos gastos totais na renda total.

O cálculo da elasticidade dos gastos com educação em relação aos gastos totais é realizado com base na estimação de $F_{i}(\ln g)$, segundo equação (2.10) abaixo (ver Gong et al. (2000)).

$\hat{F}_{i}(\ln g)=\hat{E}\left(w_{i}-\hat{\beta}_{i} x \mid \ln g\right)$

Com este intuito, primeiro se calcula o resíduo na regressão paramétrica da estimação de (2.9) e então se roda uma regressão não paramétrica Lowess unidimensional, tendo como variável dependente o erro estimado e variável explicativa o $\ln (g)$. O estimador para $F_{i}(\ln g)$ será o resultado desta última regressão. Para calcular a elasticidade total, ou de um grupo, será utilizado o gasto da família com educação como fator de ponderação.

\section{4_Resultados}

O modelo foi estimado para os gastos com educação, definidos de duas formas distintas: primeiro, consideraram-se gastos globais com educação, e segundo, os gastos diretos, definidos na seção dois.

\section{1_Resultado para gastos globais}

Após a estimação das esperanças condicionais de (2.6) e calculadas as diferenças 
entre (2.6) e (2.4), os resultados da estimação de (2.9) para toda a amostra estão descritos na Tabela 5. Também realizamos a estimação para as raças separadamente para verificar se há diferença na elasticidade de cada grupo analisado.

A variável de interesse neste artigo - raça - é estatisticamente significante (ver Tabela 5), ou seja, famílias chefiadas por pessoas que se declaram brancas possuem propensão a gastar com educação maior que o outro grupo de famílias. As variáveis filhos e anos de estudo apresentam sinal condizente com o esperado, impactando de forma positiva no aumento dos gastos com educação. Já a dummy de gênero apresentou sinal negativo nas três estimações, sugerindo que o fato de o chefe da família ser homem reduz a parcela de gastos com esse grupo de bens.

Tabela 5_ Estimação da parcela linear do modelo - Brasil Variável dependente: parcela dos gastos com educação transformada

\begin{tabular}{|c|c|c|c|c|c|c|}
\hline & \multicolumn{2}{|c|}{ Total } & \multicolumn{2}{|c|}{ Brancos } & \multicolumn{2}{|c|}{ Negros/pardos } \\
\hline & $\beta_{\mathrm{i}}$ & erro+ & $\beta_{\mathrm{i}}$ & Erro & $\beta_{i}$ & erro \\
\hline Filhos & $0,001 * *$ & 0,37 & $0,002 * *$ & 0,76 & 0,001 & 0,36 \\
\hline Raça & $0,003 * *$ & 1,19 & - & - & - & - \\
\hline Gênero & $-0,003 * *$ & 1,32 & $-0,004 *$ & 2,31 & $-0,001$ & 1,36 \\
\hline Anos de estudo & $0,002 * *$ & 0,19 & $0,002 * *$ & 0,27 & $0,002 * *$ & 0,25 \\
\hline Centro-Oeste & $0,002^{*}$ & 1,12 & $0,004^{*}$ & 2,08 & $0,003 * *$ & 1,28 \\
\hline Nordeste & $0,005 * *$ & 0,82 & $0,006 * *$ & 1,78 & $0,004 * *$ & 0,91 \\
\hline Sudeste & $0,003 * *$ & 1,28 & $0,006 * *$ & 2,07 & 0,000 & 1,69 \\
\hline Sul & $-0,004 * *$ & 1,26 & $-0,003 * *$ & 1,81 & $-0,002$ & 1,59 \\
\hline Constante & 0,000 & 0,39 & $0,001 * *$ & 0,66 & $-0,001$ & 0,44 \\
\hline $\begin{array}{l}\text { Número de } \\
\text { observações }\end{array}$ & \multicolumn{2}{|c|}{29.864} & \multicolumn{2}{|c|}{12.427} & \multicolumn{2}{|c|}{17.437} \\
\hline Elasticidade & \multicolumn{2}{|c|}{0,99} & \multicolumn{2}{|c|}{0,99} & \multicolumn{2}{|c|}{1,01} \\
\hline
\end{tabular}

Fonte: Elaboração própria a partir da POF/IBGE. 
Em relação às dummies regionais, é esperado sinal positivo das regiões Sul e Sudeste, já que essas possuem renda e também gastos muito superiores que a região de controle (Norte). O Sudeste confirma esse resultado para toda a amostra e para os brancos, mas o Sul o contraria, sugerindo que, após controlar pelos demais fatores os gastos com educação no Sul, de fato esses não são superiores aos da região Norte, como era esperado. As dummies do Centro-Oeste e do Nordeste apresentam sinal positivo e significativo, indicando que as famílias dessas regiões destinam maiores recursos para a educação do que as familias da região Norte.

A elasticidade para esses grupos também é reportada na Tabela 5. Os resultados sugerem que tanto para a amostra toda, como para os dois grupos considerados, a elasticidade-gasto com educação é unitária, ou seja, as famílias aumentam o gasto com educação proporcionalmente com o aumento nos gastos.

O próximo passo é identificar se de fato esse perfil de gastos se mantém constante em todos os grupos de renda. Nesta

Tabela 6_Estimação da parcela linear do modelo - Faixas de Renda Variável dependente: parcela dos gastos com educação transformada

\begin{tabular}{|c|c|c|c|c|c|c|c|c|}
\hline & \multicolumn{2}{|c|}{ I } & \multicolumn{2}{|c|}{2} & \multicolumn{2}{|c|}{3} & \multicolumn{2}{|c|}{4} \\
\hline & $\beta_{\mathrm{i}}$ & erro+ & $\beta_{\mathrm{i}}$ & erro & $\beta_{\mathrm{i}}$ & Erro & $\beta_{\mathrm{i}}$ & erro \\
\hline Filhos & $0,001 * *$ & 0,41 & $0,001 * *$ & 0,49 & 0,000 & 1,02 & $0,007 * *$ & 2,03 \\
\hline Raça & 0,000 & 1,10 & 0,001 & 1,12 & 0,000 & 1,91 & $0,010^{* *}$ & 3,69 \\
\hline Gênero & $-0,001$ & 1,47 & $-0,001$ & 1,14 & $-0,002$ & 2,25 & $-0,005$ & 4,09 \\
\hline Anos de estudo & $0,001 * *$ & 0,26 & $0,001 * *$ & 0,18 & $0,002 * *$ & 0,29 & $0,003 * *$ & 0,44 \\
\hline Centro-Oeste & $0,004 * *$ & 1,62 & $0,003 * *$ & 1,37 & 0,001 & 2,21 & $0,010^{* *}$ & 3,40 \\
\hline Nordeste & $-0,001$ & 1,01 & 0,002 & 1,22 & $0,010 * *$ & 2,39 & $0,008 * *$ & 3,22 \\
\hline Sudeste & $-0,001$ & 1,75 & 0,000 & 1,57 & 0,000 & 2,44 & $0,013 * *$ & 3,60 \\
\hline$\underline{\text { Sul }}$ & $0,003 *$ & 1,88 & $-0,003 * *$ & 1,40 & $-0,003$ & 2,47 & $-0,001$ & 3,52 \\
\hline Constante & $-0,001$ & 0,39 & 0,000 & 0,42 & 0,000 & 0,73 & 0,003 & 1,07 \\
\hline $\begin{array}{l}\text { Número } \\
\text { de observações }\end{array}$ & \multicolumn{2}{|c|}{7.908} & \multicolumn{2}{|c|}{8.296} & \multicolumn{2}{|c|}{7.039} & \multicolumn{2}{|c|}{6.621} \\
\hline Elasticidade & \multicolumn{2}{|c|}{1,03} & \multicolumn{2}{|c|}{1,00} & \multicolumn{2}{|c|}{0,99} & \multicolumn{2}{|c|}{0,98} \\
\hline
\end{tabular}

Legenda: $(+)$ Erro padrão multiplicado por $1.000 ;(* *)$ e em negrito: significativo a $5 \%$; $(*)$ significativo a $10 \%$; $\mathrm{E}=$ elasticidade-gasto com educação.

Fonte: Elaboração própria a partir da POF/IBGE. 
etapa, a amostra é dividida em quatro estratos de renda, e a equação 2.9 é calculada para cada faixa. Na Tabela 6 , encontram-se os resultados dessa estimação.

Os três primeiros estratos de renda concentram 78\% das famílias, com renda domiciliar per capita média de R\$135,00 (máximo de $\mathrm{R} \$ 374,00)$; nessas faixas a variável raça não se mostrou significante, o que sugere que, para tais faixas de renda, brancos e negros/pardos apresentam um padrão de gastos com educação equivalente. Esse resultado, entretanto, inverte-se no último grupo de renda, com o coeficiente racial significante, embora muito pequeno (1\%). Ou seja, para as famílias cuja renda domiciliar per capita média é mais elevada, famílias cujo chefe se declarou branco gastam mais em educação do que o segundo grupo.

A variável gênero possui sinal negativo para a maioria dos estratos, mas não se apresenta significativa para nenhuma faixa, o que indica que o sexo do chefe da família não interfere nos gastos educacionais. Em relação ao número de filhos, os resultados demonstram que esses aumentam os gastos com educação para quase todas as faixas, sendo a exceção a terceira faixa. Um resultado esperado é que, apesar do pequeno coeficiente, o nível educacional do chefe da família demonstra ser diferente de zero, para todas as estimações, e o aumento mar- ginal na parcela dos gastos é crescente com o nível de renda.

As dummies regionais apresentam comportamento distinto entre os estratos de renda, e poucas delas são significativas. Nordeste e Centro-Oeste exibem sinal positivo e significativo para três grupos; já o Sudeste só é relevante para o último estrato de renda. Por fim, o Sul apresenta o mesmo comportamento da amostra total, com sinal positivo no primeiro grupo e negativo para os demais, sendo significativa para apenas um grupo. Esses resultados indicam que, ao analisarmos um grupo homogêneo de renda, não há diferença regional na determinação de gasto com educação.

Observa-se também na Tabela 6 a estimação da elasticidade dos gastos globais com educação em relação aos gastos totais em consumo. Para todas as faixas de renda, a elasticidade se encontra próxima da unidade, ou seja, o aumento nos gastos totais leva a uma elevação nos gastos com educação na mesma proporção.

\section{2_ Resultados para os gastos diretos com educação}

Na Tabela 7, estão apresentados os resultados para os gastos diretos com educação. A diferença nos resultados das Tabelas 5 e 7 reside basicamente no sinal das dummies regionais, uma vez que os coefi- 
cientes das demais variáveis apresentam resultados semelhantes. O Nordeste e o Centro-Oeste se mantêm com sinal positivo e significativo para as três estimações, assim como o Sul mantém a significância com impacto negativo; já a dummy do Sudeste somente é significativa para brancos e com sinal positivo.

$O$ resultado interessante dessa segunda formulação é a elasticidade-gasto com educação ser muito maior que a primeira, ou seja, quando se trata de gastos diretos com educação, um aumento de 1\% nos gastos totais eleva em mais de $1 \%$ os gastos diretos com educação. A Tabela 8 traz os resultados para a estimação da curva de Engel para os gastos diretos por faixa de renda.

A análise dos resultados da Tabela 8 sugere que mais uma vez a variável raça só é significativa para a última faixa de renda, ou seja, da mesma forma que em relação aos gastos totais com educação, somente entre os mais ricos há diferença racial na determinação dos gastos com esse grupo de bens. Assim como a variável raça, as demais apresentam o mesmo comportamento dos apresentados na Tabela 6.

Tabela 7 Estimação da parcela linear do modelo - Brasil

Variável dependente: parcela dos gastos diretos com educação transformada

\begin{tabular}{|c|c|c|c|c|c|c|}
\hline & \multicolumn{2}{|c|}{ Total } & \multicolumn{2}{|c|}{ Brancos } & \multicolumn{2}{|c|}{ Negros/pardos } \\
\hline & $\beta_{\mathrm{i}}$ & erro+ & $\beta_{\mathrm{i}}$ & Erro & $\beta_{\mathrm{i}}$ & erro \\
\hline Filhos & $0,001 * *$ & 0,33 & $0,002 * *$ & 0,65 & 0,000 & 0,34 \\
\hline$\underline{\text { Raça }}$ & $0,003 * *$ & 1,05 & - & - & - & - \\
\hline Gênero & $-0,001$ & 1,13 & $-0,001$ & 1,98 & $-0,001$ & 1,15 \\
\hline Anos de estudo & $0,002 * *$ & 0,16 & $0,002 * *$ & 0,24 & $0,002 * *$ & 0,23 \\
\hline Centro-Oeste & $0,002 * *$ & 1,02 & $0,005^{* *}$ & 1,77 & $0,003 * *$ & 1,19 \\
\hline Nordeste & $0,005^{* *}$ & 0,74 & $0,007 * *$ & 1,49 & $0,004 * *$ & 0,84 \\
\hline$\underline{\text { Sudeste }}$ & 0,000 & 1,12 & $0,004 * *$ & 1,73 & $-0,002$ & 1,49 \\
\hline Sul & $-0,004 * *$ & 1,11 & $-0,002$ & 1,49 & $-0,003 * *$ & 1,36 \\
\hline Constante & 0,000 & 0,35 & 0,000 & 0,59 & $-0,001$ & 0,41 \\
\hline $\begin{array}{l}\text { Num. de } \\
\text { observações }\end{array}$ & \multicolumn{2}{|c|}{29.864} & \multicolumn{2}{|c|}{12.427} & \multicolumn{2}{|c|}{17.437} \\
\hline Elasticidade & \multicolumn{2}{|c|}{1,13} & \multicolumn{2}{|c|}{1,15} & \multicolumn{2}{|c|}{1,10} \\
\hline
\end{tabular}

Fonte: Elaboração própria a partir da POF/IBGE. 
Tabela 8_ Estimação da parcela linear do modelo - Faixas de Renda Variável dependente: parcela dos gastos restritos com educação transformada

\begin{tabular}{|c|c|c|c|c|c|c|c|c|}
\hline & & & & & \multicolumn{2}{|c|}{ J } & \multicolumn{2}{|c|}{4} \\
\hline & $\beta_{\mathrm{i}}$ & erro+ & $\beta_{\mathrm{i}}$ & erro & $\beta_{i}$ & erro & $\beta_{\mathrm{i}}$ & erro \\
\hline Filhos & $0,001 * *$ & 0,39 & $0,001 *$ & 0,45 & $-0,001$ & 0,89 & $0,005 * *$ & 1,85 \\
\hline Raça & 0,000 & 1,04 & 0,000 & 0,95 & 0,000 & 1,59 & $0,010 * *$ & 3,32 \\
\hline Gênero & $-0,001$ & 1,43 & 0,000 & 0,96 & $-0,001$ & 1,96 & $-0,002$ & 3,58 \\
\hline Anos estudo & $0,000 * *$ & 0,25 & $0,001 * *$ & 0,15 & $0,002 * *$ & 0,24 & $0,003 * *$ & 0,40 \\
\hline Centro-Oeste & $0,005 * *$ & 1,42 & $0,003 * *$ & 1,14 & 0,000 & 2,01 & $0,010 * *$ & 3,11 \\
\hline Nordeste & 0,000 & 0,90 & $0,002 * *$ & 1,03 & $0,010 * *$ & 2,25 & $0,009 * *$ & 2,91 \\
\hline Sudeste & 0,001 & 1,65 & 0,000 & 1,35 & $-0,003$ & 2,13 & $0,006^{*}$ & 3,17 \\
\hline Sul & $0,004 * *$ & 1,73 & $-0,003 * *$ & 1,11 & $-0,003$ & 2,22 & $-0,003$ & 3,08 \\
\hline Constante & $-0,001$ & 0,35 & 0,000 & 0,36 & 0,000 & 0,66 & 0,002 & 0,97 \\
\hline $\begin{array}{l}\text { Num. de } \\
\text { observações }\end{array}$ & \multicolumn{2}{|c|}{7.908} & \multicolumn{2}{|c|}{8.296} & \multicolumn{2}{|c|}{7.039} & \multicolumn{2}{|c|}{6.621} \\
\hline Elasticidade & \multicolumn{2}{|c|}{1,18} & \multicolumn{2}{|c|}{1,04} & \multicolumn{2}{|c|}{0,99} & \multicolumn{2}{|c|}{0,95} \\
\hline
\end{tabular}

Legenda: $(+)$ Erro padrão multiplicado por $1.000 ;(* *)$ e em negrito: significativo a $5 \% ;(*)$ significativo a $10 \%$;

$\mathrm{E}=$ elasticidade-gasto com educação.

Fonte: Elaboração própria a partir da POF/IBGE.

Comparando-se a elasticidade descrita nas Tabelas 6 e 8, chega-se a resultados interessantes: primeiro, em ambos os casos, a elasticidade declina, corroborando o resultado de Hoffmann (2006), nos quais os gastos com educação são inelásticos para os mais ricos. Segundo, esse movimento é mais bem observado quando se trabalha com os gastos restritos, o que é esperado, uma vez que para esses o principal gasto com educação são as mensalidades escolares, as quais são função basicamente do número de dependentes, que não se altera com o aumento nos gastos totais.

\section{5_ Considerações finais}

Este trabalho buscou analisar a curva de Engel dos gastos com educação das famílias brasileiras utilizando uma metodologia semiparamétrica e focando na questão racial. Pelos resultados obtidos, constatase que famílias chefiadas por pessoas que se denominam brancas gastam mais com esse grupo de consumo quando é analisada a amostra total e depois de feitos todos os controles, tanto para os gastos totais como para os gastos diretos com educação. 
Já a análise parcial, separando a amostra em estratos de renda, mostrou que o fato de o chefe da família não ser branco não diminui o gasto da família com a educação de seus filhos para a maioria das faixas consideradas, exceção apenas para o último estrato. Esses resultados sugerem que, entre os mais pobres, as questões raciais não influenciam na determinação da parcela dos gastos destinados à educação de seus filhos. Já entre os mais ricos, há essa diferenciação. Uma variável que se mostrou significativa para quase todas as estimações foi o nível educacional do chefe da família, sendo seu impacto positivo e crescente com o aumento da faixa de renda. $\mathrm{O}$ fato de o chefe ser homem reduz o gasto com educação.

Observando-se as elasticidades-gastos com educação, nota-se que esta é aproximadamente unitária para os gastos totais com esse grupo de bens para a amostra to$\mathrm{da}$, assim como para as raças separadas e em todas as faixas de renda. Quando considerados os gastos restritos a matrículas escolares, a livros didáticos e a material escolar, a elasticidade continua unitária nas três primeiras estimações (Brasil, brancos, negros/pardos), assim como para as primeiras faixas de renda, mas se reduz para os últimos estratos.

Os resultados não paramétricos sugerem a existência de dominância estocás- tica de primeira e segunda ordem da renda dos brancos sobre a dos negros, ou seja, se há mais pobres entre os negros/pardos do que entre o outro grupo, o que a princípio poderia representar discriminação racial. Entretanto, quando se analisa os gastos com educação, percebe-se que, após controlar pelas demais características familiares, na média os chefes de família que se consideram brancos gastam mais com educação do que os que se consideram negros/pardos, e, como dito na introdução, o diferencial de educação tem sido apontado como o maior responsável por diferenças salariais. Assim, tal resultado teoricamente pode justificar uma renda média superior para as famílias chefiadas por brancos, visto que essas investem mais em educação.

Fortalece esta conclusão o fato de que, ao se analisar os gastos com educação por faixas de renda, nos últimos estratos da distribuição (nos quais é observado o grande diferencial de renda entre brancos e negros), as análises de regressão mostram que os chefes de família que se dizem brancos gastam de fato mais com educação do que os que se dizem negros/pardos. Tais resultados são indicativos de não existência de discriminação no mercado de trabalho, mesmo nas faixas de renda mais elevadas. 


\section{Referências Bibliográficas}

ALVES, D. Parametric and semi-parametric modeling of healthcare expenditures: a household data analysis for the city of São Paulo. In: ENCONTRO BRASILEIRO DE

ECONOMETRIA, 22., Campinas, 2000. Anais... Campinas, 2000.

ALVES, D.; DISCH, R.; EVENSON, R. The demand for food in Brazil. In: ENCONTRO BRASILEIRO DE

ECONOMETRIA, 4., 1982, Águas de São Pedro. Anais... Olinda, 1982

BHALOTRA, S.; ATTFIELD, C. Intrahousehold resource allocation in rural pakistan: A semiparametric analysis. Journal of Applied

Econometrics, v. 13, n. 5, p. 463-480, 1998.

BLUNDELL, R.; CHEN, X.; KRISTENSEN, D.

Semi-nonparametric IV estimation of shape-invariant engel curves. Econometrica, v. 75 , n. 6 , p. $1613-1669,2007$.

BLUNDELL, R.; BROWING, M.; CRAWFORD, I. A. Nonparametric Engel curves and revealed preference. Econometrica, v. 71, n. 1, p. 205-240, 2003.

BLUNDELL, R.; DUNCAN, A.; PENDAKUR, K. Semiparametric estimation and consumer demand. Journal of Applied Econometrics, v. 13, n. 5, p. 435-461, 1998.
BRITO, R. J. A.; ANDRADE, M. V.; PERPÉTUO, I. H. O. Demanda por serviços de saúde. In: SILVEIRA, Fernando Gaiger; SERVO, Luciana Mendes; MENEZES, Tatiane; PIOLA, Sérgio Francisco (Orgs.). Gasto e consumo das famílias brasileiras contemporâneas. Brasília, v. 2, 2007.

CASTRO, J. A.; VAZ, F. M. Gastos das famílias com educação. In: SILVEIRA, Fernando Gaiger; SERVO, Luciana Mendes; MENEZES, Tatiane; PIOLA, Sérgio Francisco (Orgs.). Gasto e consumo das famílias brasileiras contemporâneas. Brasília, v. 2, 2007.

DEATON A.; MUELLBAUER J. An almost ideal demand system. American Economic Review, v. 70, p. 312-326, 1980.

\section{DEATON A.; MUELLBAUER J.}

Economics and consumer behavior. Cambridge University Press, 1980b.

FERREIRA, F. H. G. Os determinantes da desigualdade de renda no Brasil: luta de classes ou heterogeneidade educacional? In: HENRIQUES, R. (Org.). Desigualdade e pobreza no Brasil. Rio de Janeiro, IPEA, 2000.
FERREIRA, F. H. G.. LEITE, P. G.; LITCHFIELD, J. A.; ULYSSEA, G. Ascensão e queda da desigualdade de renda no Brasil. Econômica, Rio de Janeiro, v. 8, n. 1, p. 147-171, jun. 2006.

GONG, X.; SOEST, A.; ZHANG P. Sexual bias and household consumption: A semiparametric analysis of Engel curve in rural China. The Institute for the Study of Labor. Bonn, 2000. (Discussion Paper, 212).

GONG, X.; SOEST, A.; ZHANG, $P$. The effects of the gender of children on expenditure patterns in rural China: A semiparametric analysis. Journal of Applied

Econometrics, v. 20, p. 509-527, 2005.

HOFFMAN, R. Elasticidades-renda das despesas e do consumo físico de alimentos no Brasil metropolitano em 1995-1996. In: SILVEIRA, Fernando Gaiger; SERVO, Luciana Mendes; MENEZES, Tatiane; PIOLA, Sérgio Francisco (Orgs.). Gasto e consumo das famílias brasileiras contemporâneas. Brasília: IPEA, v. 1, p. $197-212,2000$.

HOFFMAN, R. Transferências de renda e a redução da desigualdade no Brasil e cinco regiões entre 1997 e 2004. Econômica, Rio de Janeiro, v. 8 , n. 1 , p. 55-81, jun. 2006.
INSTITUTO BRASILEIRO DE GEOGRAFIA E ESTATÍSTICA. Pesquisa de Orçamentos Familiares, 2002-2003. Rio de Janeiro, IBGE, 2004.

KINGDON, G. G. Where has all the bias gone? Detecting gender bias in the intrahousehold allocation of educational expenditure. Oxford University, 2005.

MARINHO, E.; NOGUEIRA, J. Discriminação salarial por raça e gênero no mercado de trabalho das regiões Nordeste e Sudeste. In: ENCONTRO NACIONAL DE ECONOMIA, 34., Vitória, 2006. Anais... Vitória: ANPEC, 2006. MENEZES, T. A.; SILVEIRA, F. G.; AZZONI, C. R. Demand elasticities for food products in Brazil: a two-stage budgeting system. Applied Economics, v. 40, p. $2557-2572,2008$.

MICHALEK, J.; KEYZER, M.

Estimation of a two-stage Les-Aids consumer demand system for eight EC countries. European Review of Agricultural Economics, n. 19, p. $137-163,1992$.

ROBINSON, P. Root-N-consistent semiparametric regression. Econometrica, v. 56, p. 31-954, 1988. 
ROZELLE, S.; GIBSON, J. How elastic is calorie demand? Parametric, nonparametric, andsemiparametric results for urban Papua New Guinea. Califronia Agriculturar Experiment Station, 2000. (Working Paper, n. 00-022).

SCHWARTZMAN, S. Educação: a nova geração de reformas. In: GIAMBIAGI, Fábio; REIS, José Guilherme; URANI, Andréi

(Orgs.). Reformas no Brasil: balanço e agenda, Rio de Janeiro: Nova Fronteira, 2004. p. 481-504.

SOARES, S. Distribuição de renda no Brasil de 1976 a 2004 com ênfase no periodo entre 2001 e 2004. Brasília: IPEA, fev. 2006a. (Textos para Discussão, 1166).

SOARES, S. Análise de bem-estar e decomposição por fatores da queda na desigualdade entre 1995 e 2004. Econômica, Rio de Janeiro, v. 8, n. 1, p. $83-115$, jun. 2006 b.

SOARES, S. S. D. O perfil da discriminacão no mercado de trabalhohomens negros, mulheres brancas e mulheres negras. IPEA, 2000.

(Texto para discussão, 769).

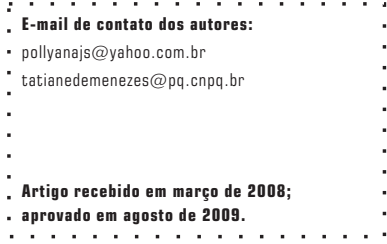


Tabela A1_ Cinco grandes grupos dos gastos com educação e alguns itens de cada grupo

\begin{tabular}{|c|c|c|}
\hline Classificação I & Classificação II & Descrição \\
\hline \multirow{4}{*}{ Cursos regulares } & Pré-escola & Curso pré-escolar \\
\hline & Ensino fundamental & Curso regular de primeiro grau \\
\hline & Ensino médio & Curso regular de segundo grau \\
\hline & Ensino superior & Curso regular de terceiro grau \\
\hline \multirow{5}{*}{ Outros cursos } & Pós-graduação & Cursos de mestrado e doutorado \\
\hline & Cursos de idiomas & Cursos de inglês, alemão, francês, etc. \\
\hline & Cursos de treinamento & $\begin{array}{l}\text { Cursos de informática, datilografia, dança, desenho, } \\
\text { culinária, profissionalizantes, etc. }\end{array}$ \\
\hline & $\begin{array}{l}\text { Cursos de esporte } \\
\text { e lazer }\end{array}$ & $\begin{array}{l}\text { Cursos de ginástica, hidroginástica, balé, ioga, } \\
\text { escolinhas de futebol, natação, etc. }\end{array}$ \\
\hline & Outros cursos & $\begin{array}{l}\text { Curso pré-vestibular, creche, supletivo, extensão, } \\
\text { educação a distância, etc. }\end{array}$ \\
\hline $\begin{array}{l}\text { Livros didáticos } \\
\text { e revistas técnicas }\end{array}$ & $\begin{array}{l}\text { Livros didáticos } \\
\text { e revistas técnicas }\end{array}$ & $\begin{array}{l}\text { Livros didáticos e revistas técnicas, apostilas, } \\
\text { dicionários, etc. }\end{array}$ \\
\hline Artigos escolares & Artigos escolares & $\begin{array}{l}\text { Cadernos, lápis, borracha, tesoura, cola, régua, } \\
\text { compasso, giz, pastas, mochilas, etc. }\end{array}$ \\
\hline Taxas e contribuições & Taxas e contribuições & $\begin{array}{l}\text { Taxas escolares, inscrições em seminários e } \\
\text { congressos, emissão de diploma, carteira de } \\
\text { estudante, associação de pais e mestres, matrícula } \\
\text { escolar, multa de biblioteca, etc. }\end{array}$ \\
\hline Outros gastos & Outros gastos & $\begin{array}{l}\text { Aulas particulares, cursos em discos ou fita cassete, } \\
\text { uniformes escolares, tradutores, excursões, } \\
\text { formaturas, etc. }\end{array}$ \\
\hline
\end{tabular}

Fonte: Castro e Vaz (2007). 
